\title{
AVALIAÇÃO DO PERFIL DE POTENCIALIDADES E NECESSIDADES DE CRIANÇAS EM RESOLUÇÃO DE PROBLEMAS MATEMÁTICOS
}

\author{
EVALUATION OF THE PROFILE OF POTENTIALITES NECESSITIES CHILDRENS IN \\ RESOLUTION OF MATHEMATICAL PROBLEMS
}

GRAZIELLA RIBEIRO SOARES MOURA ${ }^{l}$

\begin{abstract}
RESUMO: Este estudo teve como objetivo realizar uma avaliação do perfil de potencialidades e necessidades de crianças em idade escolar sobre resolução de problemas matemáticos, com o propósito posterior de direcionar um programa de intervenção que aprimore as condições de aprendizagem dessas crianças. O estudo foi realizado em uma escola particular do município de Bauru (São Paulo). Foram aplicados dois protocolos para medir as capacidades de 38 crianças com 10 anos de idade cursando a $4^{\mathrm{a}}$ série do ensino fundamental. $\mathrm{O}$ primeiro protocolo avaliou a resolução de problemas de matemática pelos estudantes e as dificuldades encontradas nesta tarefa. O segundo protocolo analisou as capacidades linguiísticas das crianças (leitura e compreensão de textos lingüísticos). Em seguida foi realizada uma análise deste material que possibilitou a identificação dos elementos presentes e ausentes necessários à prática de resolução de problemas, bem como uma correlação entre a compreensão de textos e a compreensão de enunciados matemáticos. Os resultados indicaram que compreender bem textos de natureza lingüística não significa compreender bem textos matemáticos. Percebeu-se também que o repertório para a resolução de problemas simples destes estudantes era bom, mas há necessidade de os professores utilizarem problemas diversificados e mais complexos em sala de aula.
\end{abstract}

Palavras-chave: Educação. Educação Matemática. Resolução de problemas matemáticos. Avaliação.

\begin{abstract}
This study it had as objective to carry through an evaluation of the profile of potentialities and necessities of children in pertaining to school age on resolution of mathematical problems, with the posterior intention to direct an intervention program that improves the conditions of learning of these children. The study it was carried through in a particular school of the city of Bauru (São Paulo). Series of basic education had been applied two protocols to measure the capacities of 35 children with 10 years of age being attended a course $4^{\mathrm{a}}$. The first protocol evaluated the resolution of problems of mathematics for the students and the difficulties found in this task. As the protocol analyzed the linguistic capacities of the children (reading and understanding of linguistic texts). After that an analysis of this material was carried through that made possible the identification of the elements necessary gifts and absentees to the practical one of resolution of problems, as well as a correlation it enters the understanding of texts and the understanding of mathematical statements. The results had indicated that to understand texts of linguistic nature well it does not mean to understand mathematical texts well. It was also perceived that the repertoire for the resolution of simple problems of these students was good, but has necessity of the professors to use diversified and more complex problems in classroom.
\end{abstract}

Keywords: Education. Mathematical education. Resolution of mathematical problems. Evaluation.

\footnotetext{
${ }^{1}$ Pedagoga, mestre em Educação para a Ciência (Unesp-Bauru) e doutora em Educação Especial (UFScar). Professora do Instituto de Ensino Superior de Bauru e da rede pública do estado de São Paulo. gribeiro001@ig.com.br
} 


\section{INTRODUÇÃO}

A aprendizagem é um domínio inerente ao ser humano. As condições neurológicas, sociais e psicológicas da pessoa quando em harmonia favorecem adequadamente a aprendizagem.

Para Carraher et al (1995) a aprendizagem da matemática na sala de aula é um momento de interação entre a matemática organizada pela comunidade científica, ou seja, a matemática formal e a matemática como atividade humana. A matemática praticada na sala de aula é uma atividade humana porque o que interessa nessa situação é a aprendizagem do aluno.

Atualmente é inegável a importância de os estudantes serem capazes de solucionar problemas. Atividades de resolução de problemas são extremamente úteis, pois aprimoram o pensamento, o raciocínio lógico, a autonomia, a capacidade de enfrentamento de situações adversas, o desenvolvimento da habilidade de criar estratégias para diversas circunstâncias. Nesse sentido, atividades de resolução de problemas são altamente produtivas nas escolas e os professores necessitam, não só oferecer situações-problema como também, instigar os estudantes a desejarem alcançar a solução das situações propostas, encorajando-os a buscar caminhos para a solução.

Os estudos de Dochrell e McShane (2000) revelam que as crianças freqüentemente tratam as operações aritméticas como mera manipulação de símbolos, sem relacionar os números aos conceitos de quantidades a que se referem. Em muitas crianças, a falha matemática não se encontra na ausência das habilidades básicas e sim na capacidade de relacionar essas habilidades com o mundo. A habilidade de manipulação de números não tem um fim em si mesmo. O poder real dos números é a possibilidade de representação dos problemas da vida diária em linguagem matemática para propósitos de cálculo. As crianças freqüentemente apresentam dificuldades em representar os problemas quantitativos do mundo real de forma matemática, passo principal necessário para resolver problemas, principalmente com enunciados escritos.

Os problemas matemáticos com enunciados escritos são comuns nas salas de aula. Normalmente descrevem situações em que as quantidades são manipuladas. São usados com o propósito de unir a prática do cálculo aritmético com situações em que a aritmética pode ser aplicada.

Segundo Polya (1994) para resolver problemas a criança precisa primeiramente entender a situação proposta, em seguida planejar um método de resolução, executar esse plano e, em seguida, verificar se o plano respondeu ao problema proposto. A compreensão é baseada em habilidades cognitivas e lingüísticas da criança, o planejamento envolve a construção de uma 
representação matemática do problema, a execução do plano significa a realização de uma ou mais estratégias e procedimentos matemáticos previamente selecionados e a verificação é uma avaliação que a pessoa faz para reconhecer se a sua estratégia resolveu a situação problemática.

Para Echeverria e Pozzo (1998) as estratégias são importantes, mas não as principais habilidades para se conseguir resolver problemas. Esta prática depende, também, da maneira como a estrutura se adapta à tarefa e da presença de regras, algoritmos e operadores concretos, isto é, técnicas que possibilitam o desenvolvimento mais efetivo dos planos de resolução.

Os estudo de Santos (1998) revelam que a pergunta do problema pode ser diretamente traduzida em adição ou subtração. No entanto, quando não há uma tradução direta, as crianças apresentam dificuldades maiores, como no exemplo a seguir: Sema tinha algumas fotos de artistas de cinema. Na semana passada, foi até a banca de seu Sebastião e comprou mais 17 fotos ficando com 42. Quantas fotos Selma tinha antes? ( Mudança - Série Inicial Desconhecida - situação de aumento) $)^{2}$.

Neste exemplo, as duas quantidades devem ser subtraídas para se obter a resposta, mas o problema não especifica que se trata de uma subtração e ainda indica a palavra "mais". Assim, a criança deve traduzir o problema em uma representação que combina as duas quantidades para se obter a inicial. Este exemplo ilustra a relação relativamente complexa que pode existir entre a forma verbal de um problema e sua representação matemática.

As dificuldades em resolução de problemas podem aparecer na compreensão do problema, na construção de um modelo matemático ou na execução de estratégias de resolução dos problemas com enunciados escritos. Entretanto, os principais determinantes do desempenho da criança parecem ser a complexidade do texto e a disponibilidade de bases para a representação matemática do problema.

Segundo Mayer (1983), a compreensão do enunciado matemático é o primeiro passo para a sua solução. Para compreender a questão a pessoa precisa traduzir a linguagem expressa em informações matemáticas e isto requer conhecimentos lingüísticos, semânticos e esquemáticos que facilitem a compreensão da tarefa, permitem a sua representação em termos matemáticos e ajudem a elaborar um plano para a resolução.

Outro ponto importante para o sucesso em resolução de problemas está no fato de que esses dados expressos lingüística e matematicamente sejam relacionados com os conceitos e idéias que estão armazenados na memória da pessoa. Essa relação permite que a informação inicial seja transformada em uma informação que a pessoa possa usar (MAYER, 1983). Se a

\footnotetext{
${ }^{2}$ Classificação de tipos de problemas elaborada por Carpenter e Moser (1982).
} 
pessoa não tiver informações como, por exemplo, de cálculos aritméticos presentes na memória, dificilmente conseguirá elaborar esquemas e representações mentais suficientes para resolver o problema.

A resolução de problemas exige a disponibilidade, na memória de uma estrutura apropriada para representar o problema. A questão verbal deve ser traduzida dentro dessa estrutura. Algumas crianças podem não ter o conhecimento lingüístico e conceitual necessário para entender a situação expressa.

O uso de representações mentais é imprescindível para o processo de resolução de problemas, assegura Vieira (1999). A abordagem do processamento da informação desafia a pessoa cognitivamente, possibilitando um conhecimento mais aprofundado da atividade de resolução de problemas matemáticos e, sem dúvida, oferece ferramentas para a construção mais eficaz da aprendizagem e capacidade para a resolução. Durante a resolução de problemas matemáticos, a pessoa pode utilizar dois tipos de pensamento: o produtivo e o reprodutivo (WERTHEIMER, 1945). Quando a pessoa produz novas soluções partindo de uma organização ou reorganização das informações do problema possui um pensamento produtivo, ao passo que, quando apenas recorre às regras aprendidas de forma mecânica, sem reflexão, aplicando métodos já conhecidos está apenas reproduzido fórmulas apresentando um pensamento reprodutivo.

Muitas vezes as pessoas só reproduzem conhecimentos ao resolver problemas. Isto pode ser evidenciado quando utilizam palavras-chave para decifrar as informações dadas na situação. Figueiredo (1985) e Santos (1998) realizaram alguns estudos e perceberam que a utilização de palavras-chave pode encaminhar equivocadamente o pensamento da pessoa. Esse fato foi evidenciado quando as crianças investigadas associavam palavras como ganhou a operações de adição; vendeu, a operações de subtração e nem sempre estes vocábulos se referem a conceitos aditivos e subtrativos.

Nesse sentido, a meta principal da escola, em relação ao ensino da matemática, seria tentar estabelecer uma ponte entre os símbolos e as situações em que estes símbolos são representados, favorecendo o desenvolvimento da compreensão dos conceitos matemáticos. (SELVA, 1983).

Os estudos de Nunes e Bryant (1987) revelam que muitas crianças se saem bem com relação à aprendizagem da matemática cotidiana, ou seja, no dia-a-dia conseguem lidar muito bem com o cálculo e as situações-problema, porém, quando devem transferir essa linguagem para a matemática formal escolarizada, sentem dificuldades. 
Professores e estudantes devem atribuir atenção especial à linguagem e à linguagem matemática para que a interpretação de enunciados matemáticos ocorra satisfatoriamente. Garnica (1992) afirma que tanto textos curtos como longos expressos nos problemas matemáticos devem ser compreendidos para serem resolvidos.

Malta (2003) também enfoca a importância da linguagem no processo de aprendizagem em matemática. Discute a idéia de que os alunos de hoje, pela falta do exercício da leitura ainda não perceberam que entender não é apenas identificar o que está sendo lido com algo já conhecido anteriormente; não descobriram que o entendimento de um texto não trivial é o resultado de um processo de construção, processo no qual construímos objetos mentais que vão dar significados aos novos conceitos (ou situações) que estão sendo apresentados. Na leitura de um texto literário, conduzido pelo autor, vai-se construindo personagens (mentais), cenários e paisagens que darão sentido à história criada pelo autor. Na leitura de um texto matemático, temos que construir, também guiados pelo autor, objetos mentais que darão significados aos conceitos abstratos (e suas relações) que estão sendo introduzidos sendo que, para um aluno, esse processo de construção passa pela interação com o professor.

Reconhecendo a importância da aprendizagem de resolução de problemas matemáticos esta pesquisa procurou realizar uma avaliação em crianças de $4^{\mathrm{a}}$ série do ensino fundamental com e sem dificuldades em resolução de problemas matemáticos com o propósito de identificar seu perfil de potencialidades e necessidades. Essa avaliação poderá sugerir elementos para a construção posterior de um programa de intervenção capaz de melhorar a aprendizagem dessas crianças, bem como material teórico-metodológico de orientações para o trabalho docente.

De acordo com Dochrell e McShane (2000), a avaliação é um processo de coleta de informações para um objetivo específico. Trata-se de um processo de direcionamento da tomada de decisões sobre uma criança, identificando seu perfil de capacidades e necessidades. A avaliação deve estar voltada para as hipóteses levantadas e estas hipóteses devem ser baseadas na compreensão da criança e nos componentes cognitivos, dificuldades de aprendizagem, bem como na análise do meio onde a criança aprende naquele momento.

O processo de avaliação poderá detectar os repertórios que essas crianças possuem e não possuem que promovem a capacidade de resolver situações problemas.

\section{Método}

A pesquisa foi realizada em uma escola particular de Ensino Fundamental do município de Bauru - SP. Inicialmente a pesquisadora manteve contato com a equipe escolar e após 
autorização das instituições e dos pais, aplicou uma avaliação em todos os alunos de $4^{\mathrm{a}}$ série de uma classe dessa escola, durante o período em que estavam em aulas. Para esta avaliação foram usados três protocolos com exercícios.

Os participantes foram crianças com 10 anos, entre meninos e meninas que cursavam a $4^{\mathrm{a}}$ série do ensino fundamental. Foram avaliados 35 alunos do período vespertino sendo que três desses alunos, segundo as professoras da sala, apresentavam dificuldades de aprendizagem em matemática. Todos os alunos dessas classes foram avaliados durante o período normal de aulas.

Vale ressaltar que dois dias antes do início da coleta de dados, a pesquisadora esteve na escola, como de costume, pois este colégio já era local de freqüência da pesquisadora, durante o horário de recreio dos alunos para que houvesse maior familiarização entre pesquisadora e participantes.

A coleta de dados foi realizada em cinco sessões de 5 a 22 de outubro de 2004. Durante a realização da atividade a pesquisadora tomou alguns cuidados como: 1 . observar se as crianças não copiavam a resposta do colega. Com relação a essa questão, nenhuma criança realizou esse comportamento. Todos resolveram seus problemas sozinhos. 2. Tempo médio de execução dos problemas. As crianças levaram, em média, dez minutos para resolver quatro situações-problema. Pode-se perceber que o tempo de latência para realização da atividade foi absolutamente normal para a idade e o nível cognitivo das crianças.

Após conversar em sala de aula com os participantes, foi aplicado o primeiro teste que se constituiu de um protocolo com quatro situações-problema escolares de tipos diversos seguindo situações conhecidas e segundo a classificação de Carpenter e Moser (1982): a) Em uma escola de Bauru existem 26 alunos que estudam pela manhã na $4^{a}$ série e 33 que estudam à tarde. Quantos alunos, ao todo, estudam na $4^{a}$ série desta escola? (Combinação - série desconhecida); b) Rosângela fez 23 bandeirinhas de São João e sua irmã Rose fez 19 a mais do que ela. Quantas bandeirinhas Rose fez? (Comparação - quantidade maior desconhecida - termo-a-termo); c) $\mathrm{Na}$ $4^{a}$ série tem 45 cadeiras e 33 crianças. Quantas cadeiras eu preciso retirar da sala para ficar com a mesma quantidade de crianças? (Igualização - decréscimo na quantidade maior).; d) Laura tinha 20 papéis de carta e ganhou alguns de sua prima ficando com 31. Quantos papéis de carta ela ganhou? (Mudança - transformação desconhecida - situação de acréscimo).

O segundo protocolo utilizado foi o de compreensão de leitura de três textos de tipos diferentes: um narrativo, um descritivo e um informativo. Após a leitura, individual, as crianças responderam a algumas questões escritas relacionadas aos textos, também em situação de sala de 
aula. Não houve cópia, nem comentários entre os participantes com relação às respostas das questões.

Posteriormente, em dias diferentes, a pesquisadora avaliou outras habilidades relacionadas a problemas matemáticos, individualmente em uma sala de aula desocupada naquele horário. Para garantir que não houvesse prejuízo intelectual aos alunos, a pesquisadora sugeriu à professora que estipulasse os melhores dias e horários para que não atrapalhasse a aprendizagem dos alunos. A professora, então, escolheu alguns momentos em que ela estaria revisando conteúdos ou aplicando exercícios os quais já eram do conhecimento dos participantes.

Para esta avaliação foi utilizado um terceiro protocolo elaborado pela pesquisadora que verificou as seguintes habilidades: 1. Leitura fluente - as crianças leram um dos textos em voz alta para a pesquisadora; 2 . Operações aritméticas - os participantes resolveram várias operações aritméticas (adição, subtração, multiplicação e divisão); 3. Resolução de problemas orais - este protocolo continha três situações-problema que a pesquisadora fez oralmente para que as crianças resolvessem, sem a utilização do papel e lápis. Utilizou-se para esses problemas a mesma classificação dos problemas escritos, porém, com enunciados diferentes: a) Marcos tinha 43 tazos e num jogo perdeu 13. Com quantos tazos ele ficou? (Mudança - resultado desconhecido) situação de decréscimo); b) Paulo tinha 12 chocolates e comeu alguns ficando com 7. Quantos chocolates ele comeu?( Mudança -transformação desconhecida - situação de decréscimo); c) Karla fez 23 bombons para seu aniversário e sua irmã fez 19 a menos do que ela. Quantos bombons a irmã de Karla fez?

Durante a coleta de dados a pesquisadora observou e registrou, também, o nível de memória, atenção e concentração dos alunos nas atividades propostas.

\section{RESULTADOS E DISCUSSÃO}

Os dados foram tabulados utilizando-se a forma de registro de porcentagem, conforme demonstra a tabela. O total de participantes foi de 35 .

Tabela 1 - Porcentagem de habilidades dos participantes

Leitura fluente $=74,8 \%$

Leitura não fluente $=25,2 \%$

Boa compreensão de leitura $=98,3 \%$ 
Nível médio de compreensão de leitura $=1,7 \%$

Realização de operações aritméticas $=100 \%$

Resolução de problemas escritos $=100 \%$

Resolução de problemas orais $=88,6 \%$

Boas memória, atenção e concentração $=48,6 \%$

Nível médio de memória, atenção e concentração =51,4\%

O gráfico a seguir ilustra os dados obtidos mediante o estudo:

\section{Habilidades dos participantes}

\begin{tabular}{|c|c|}
\hline & $\begin{array}{l}\square \text { leitura fluente } \\
\square \text { leitura não fluente }\end{array}$ \\
\hline $100 \%$ & $\square$ compreensão de leitura \\
\hline $\begin{array}{l}90 \% \\
80 \%\end{array}$ & $\begin{array}{l}\square \text { compreensão de leitura } \\
\text { média }\end{array}$ \\
\hline $\begin{array}{l}70 \% \\
60 \%\end{array}$ & $\begin{array}{l}\text { - operações aritméticas } \\
\text { realizadas }\end{array}$ \\
\hline $50 \%$ & $\begin{array}{l}\square \text { resolução de problemas } \\
\text { escritos realizada }\end{array}$ \\
\hline $\begin{array}{l}40 \% \\
30 \%\end{array}$ & $\begin{array}{l}\square \text { resolução de problemas } \\
\text { orais realizada }\end{array}$ \\
\hline $20 \%$ & $\begin{array}{l}\square \text { memória, atenção e } \\
\text { concentração boas }\end{array}$ \\
\hline $\begin{array}{l}10 \% \\
0 \%\end{array}$ & $\begin{array}{l}\text { memória, atenção e } \\
\text { concentração médias }\end{array}$ \\
\hline
\end{tabular}

Gráfico 1 - Perfil de habilidades referentes à resolução de problemas matemáticos 
A leitura e sua compreensão são habilidades que fazem parte do repertório lingüístico necessário à resolução de problemas. Conforme assinalado por Mayer (1983), é preciso, após a leitura do problema, que a pessoa compreenda a mensagem expressa em um sistema da língua materna e represente-a de forma matemática. Para que isto ocorra é imprescindível a existência de um conhecimento lingüístico, semântico e esquemático. Além disso, para resolver um problema, a pessoa precisará possuir, em sua memória, algumas informações matemáticas como regras, algoritmos e esquemas.

Os dados demonstraram que as crianças investigadas possuem essas informações matemáticas as quais podem ser nomeadas de "modelo matemático" e se recorrem a esse modelo (preservado na memória) no momento de resolver a situação, seja ele oral ou escrito, pois tanto nos problemas escritos quanto nos orais isso foi revelado.

As crianças que não tinham leitura fluente conseguiram resolver normalmente os problemas escritos. Uma dessas crianças apresentou uma leitura absolutamente não fluente, no entanto, resolveu corretamente todos os problemas propostos. Não se pode, portanto, afirmar enfaticamente que para se compreender uma situação-problema de matemática é necessário ler o enunciado fluentemente. Este fato pode representar que a leitura fluente, embora pareça ser uma habilidade necessária para a resolução de problemas matemáticos não influenciou decisivamente nas atividades.

Com relação à compreensão de leitura, conforme verificado na tabela, os participantes obtiveram grande êxito. Assim, é possível notar, conforme Mayer (1983) que as crianças conseguiram traduzir a linguagem expressa no problema em conhecimentos, informações e idéias matemáticas, pois todas possuíam um repertório bom de capacidades para resolução de operações aritméticas (100\%).

Sobre este fato, é importante ressaltar que, ao transferir a linguagem escrita em processos matemáticos fundamentais para a resolução de problemas, elas preocupam-se com as palavraschave do enunciado, ou seja, ao ler ou ouvir a situação-problema, os alunos fixam seu pensamento nos numerais apresentados e nas palavras "guias" como: "a irmã dela fez 19 anos a menos - menos, então tenho que tirar", “comeu 7 - é de menos”. Isso confirma o que Figueiredo (1985) e Santos (1998) pontuam, que muitas crianças, ao resolverem um problema escolar, escolhem as palavras-chave para obter a resposta, o que evidencia uma prática prejudicial, pois dessa forma não raciocinam, não interferem, não constroem o pensamento matemático produtivo conforme assinala Wertheimer (1945) e sim realizam um exercício mecanicamente. 
Os resultados revelaram que as crianças com 10 anos conseguem resolver bem os problemas do tipo exercício denominados escolares, conforme se vê na tabela. Dos 35 alunos, 21 acertaram as quatro situações-problema propostas e os demais erraram apenas um problema. Somente uma criança errou dois. O problema que demonstrou maior dificuldade foi o problema $b$ que propõe uma situação de comparação - quantidade maior desconhecida - termo a mais, confirmando o que assinala Santos (1998) quando relata que o tipo de problema mais difícil é aquele em que é preciso comparar quantidades.

O mesmo acontece com os problemas orais quando a maior dificuldade está no problema do mesmo tipo (letra c - 12 crianças erraram este problema). Foi possível perceber que não existe correlação entre saber resolver problemas escritos e orais, pois $11,4 \%$ dos alunos que não realizaram os problemas orais, realizaram os problemas escritos. Também percebeu-se que $91,4 \%$ das crianças que apresentaram alguma dificuldade em realizar problemas escritos realizaram satisfatoriamente os três problemas orais. Este fato sugere que a linguagem escrita é mais difícil de ser compreendida do que a linguagem oral (LIMA, 2002).

O problema oral mais difícil teve um tempo de latência maior por dois motivos: primeiro, porque a compreensão e a capacidade de representação mental são menores e, segundo, porque cerca de $50 \%$ das crianças procuram resolver a operação dos problemas utilizando o algoritmo da compensação ou do empréstimo, por exemplo, para realizar a subtração 23 -19 faz: 9 para 13 (porque emprestou uma dezena do 2), dá 4, devolve uma dezena para o 1 somando-se 2, então, 2 para chegar no 2 é igual a 0 , resposta 4; o que torna a resposta mais demorada, pois processa o algoritmo mentalmente.

Cerca de $50 \%$ dos alunos resolveram a operação subtrativa por meio da idéia aditiva da subtração, por exemplo: 7 para chegar no 12 é igual a 5. Este algoritmo facilitaria a resolução da subtração 23 - 19, mas nem todas as crianças possuem essa representação.

As estratégias utilizadas para resolução de problemas são importantes, conforme afirma Echeverria e Pozo (1998). Com relação aos problemas escolares solicitados verificou-se que as crianças possuíam uma estratégia bastante simplista que se pauta na montagem vertical de uma operação e sua resolução por meio de um algoritmo.

As crianças indicadas como aquelas com dificuldades de aprendizagem apresentaram o seguinte desempenho: com relação aos problemas orais, duas delas acertaram os três problemas propostos. Quanto à compreensão de leitura dos textos não matemáticos estes três alunos obtiveram bom êxito. Dessa forma não se pode afirmar que a boa compreensão de leitura de textos é fator decisivo para compreensão de resolução de problemas matemáticos, pois 
compreenderam bem os textos narrativo, descritivo e informativo, mas apresentaram dificuldade na leitura e compreensão dos enunciados matemáticos.

As crianças quando em situação de pesquisa apresentam boa concentração e atenção. Quanto à memória percebeu-se que 51,4\% solicitaram que a pesquisadora repetisse a situação lida, principalmente os numerais expressos nos problemas orais. A memória é um fator importante para o processo de aprendizagem (FONSECA, 1995). A idéia é que os professores pratiquem mais problemas orais em sala para estimular a memória dos alunos.

No último dia a pesquisadora aplicou, por escrito, um problema para todos os alunos em sala. A situação proposta foi a seguinte: Sérgio possui nove bolinhas do mesmo tamanho e da mesma cor. Dessas bolinhas, oito têm a mesma massa e uma tem massa maior do que as demais. Como Sérgio pode descobrir qual é a Bolonha de maior massa utilizando uma balança de dois pratos e fazendo apenas duas pesagens? $?^{3}$

Nenhuma criança conseguiu resolver essa situação. Este problema evidenciou ausência de ativação de esquemas conceituais e representações mentais (MALTA, 2003, VIEIRA, 1999, MAYER, 1983). Esta questão pode parecer difícil por se tratar de um problema diferente daquele com os quais os estudantes estão acostumados e porque está distante do seu cotidiano, mas se as crianças forem submetidas desde pequenas a esse tipo de raciocínio e apreenderem os conceitos necessários para se chegar à resposta, certamente, aos dez anos, serão capazes de realizar este desafio. É fundamental que os alunos tenham contato com situações que expressam elementos de seu mundo real para melhor aprendizagem (DOCHRELL; MCSHANE, 2000).

Este tipo de problema demonstrou que as crianças não apresentavam um pensamento produtivo e sim reprodutivo. Neste caso, elas procuraram reproduzir os conhecimentos matemáticos que tinham, mas que não eram suficientes e aplicáveis nesta situação a qual exigia produção de conhecimentos matemáticos, relações matemáticas, construção e ativação de esquemas e representações mentais, associadas aos símbolos e conceitos expressos.

Isso evidencia que as crianças não possuíam estratégias de resolução, que segundo Echeverria e Pozo (1998) e Polya (1994) são altamente instrutivas. Normalmente as crianças só reproduzem situações de operações aritméticas aprendidas, por isso se saem bem em problemas escolares os quais são chamados de exercícios (citados anteriormente).

É necessário que os professores desenvolvam habilidades estratégicas nas crianças para melhor desempenho nestas situações.

\footnotetext{
${ }^{3}$ DANTAS, S.; VIEIRA, F.; RIBEIRO, J. A escola é nossa (4 $4^{a}$ série). São Paulo: Scipione, 2003.
} 
A pesquisadora questionou as crianças quanto a gostar ou não de matemática. Do total, $51,5 \%$ afirmaram gostar dessa ciência, mas foi verificado que não existe correlação entre gostar da matemática e realizar bem os exercícios, pois dentre esses $51,5 \%$ que gostam da matemática estão aqueles que apresentaram alguma dificuldade e entre os 48,5\% que disseram não gostar ou gostar mais ou menos estão os estudantes que têm cálculo rápido, correto e bom desempenho.

\section{CONSIDERAÇÕES FINAIS}

A pesquisa revelou que as crianças com 10 anos, possuem um grande potencial para resolução de problemas matemáticos do tipo escolares. Elas são capazes de compreender a mensagem expressa lingüisticamente e transferir em termos matemáticos sejam orais ou escritos, mas suas potencialidades podem ser aumentadas a partir de sugestões problemas diferentes das situações apresentadas.

Os alunos investigados apresentaram o seguinte repertório de habilidades necessárias para resolução de problemas: informações matemáticas (uso de regras, algoritmos aritméticos, símbolos), boa leitura e compreensão, os quais podem ser aprimorados.

Foi possível inferir que as suas necessidades estão no âmbito de um contato maior com situações-problema que requeiram a utilização do pensamento produtivo que os façam, além de compreender a situação, elaborar um plano, executá-lo e verificar sua procedência.

Nesse sentido, é possível salientar um melhor direcionamento, por parte dos docentes, quanto à aprendizagem de resolução de problemas matemáticos. A sugestão é que os professores se preocupem em oferecer aos estudantes situações diversificadas de problemas que exijam o uso do raciocínio e de um pensamento matemático capaz de produzir estratégias e a busca de soluções ainda não existentes.

Normalmente a escola ensina os conteúdos da matemática escolar e posteriormente aplica nos alunos uma série de problemas para resolverem. Uma idéia seria inverter o procedimento pedagógico, ou seja, partindo de situações-problema os professores ensinariam os conteúdos matemáticos. Essa metodologia, talvez, auxiliaria os estudantes a entender os problemas como algo inerente à vida cotidiana, pois estariam contextualizados, reduzindo a noção de que problemas são exercícios meramente mecânicos.

Pesquisas nessa direção podem trazer muitas orientações para a prática pedagógica e maximizar a aprendizagem matemática dos estudantes. 


\section{REFERÊNCIAS BIBLIOGRÁFICAS}

CARPENTER, T., P.; MOSER, J., M. The Development of Addition and Subtraction ProblemSolving Skill. In: CARPENTER, J. MOSER, J.; ROMBERG, T. (Orgs.). Addition and Subtracion: a Cognitive Perspective. Hillsdale, NJ: Erlbaum, p. 9-24, 1982.

CARRAHER, T..N.; et al. Na vida dez, na escola zero.10. ed. São Paulo: Cortez, 1995.

DOCKRELL, J.; MCSHANE, J. Crianças com dificuldades de aprendizagem: uma abordagem cognitiva. Porto Alegre: Artes Médicas, 2000.

ECHEVERRÍA, M., D. P., P.; POZO , J., I. Aprender a resolver problemas e resolver problemas para aprender. In: POZO, J., I. (Org.) A solução de problemas: aprender a resolver, resolver para aprender. Porto Alegre: Artmed, 1998.

FIGUEIREDO, A. M. C. Resolução de problemas de matemática na escola de $1^{o}$ grau e o uso de "palavras-chaves" como método de ensino. 1985. Dissertação (Mestrado em Educação), Faculdade de Educação, Recife: UFPE.

FONSECA, V. da. Dificuldades de aprendizagem. 2.ed. Porto Alegre: Artes Médicas, 1995.

GARNICA, A. V. M. A interpretação e o fazer do professor de matemática: um estudo sobre a possibilidade do exame hermenêutico na Educação matemática. 1992. Dissertação (Mestrado em Educação Matemática), Faculdade de Educação, Universidade Estadual Paulista, Rio Claro.

LIMA, E. S. Quando a criança não aprende a ler e a escrever. São Paulo: Sobradinho 107, 2002.

MALTA, I. Sobre um método não tradicional para aprender cálculo. In: CARVALHO, L.M.; GUIMARÃES, L. C. (Org.). História e Tecnologia no Ensino de Matemática, vol. 1. Rio de Janeiro: IME-UERJ, p. 179-186, 2003.

MAYER, R. E. Thinking, problem solving, cognition. New York: W. H. Freeman and Company, 1983.

NUNES, T; BRYANT, P. Crianças fazendo matemática. Porto Alegre: Artes Médicas, 1997.

POLYA G. A arte de resolver problemas: um novo enfoque do método matemático. Rio de Janeiro: Interciência, 1994 (original publicado em 1975). 
SANTOS, R. B. dos. Investigando contextos de utilização de materiais concretos como auxiliares na resolução de problemas matemáticos com estruturas aditivas. 1998. Dissertação (Mestrado em Educação). Faculdade de Educação, UFPE, Recife.

SELVA, A .C. V. A influência de diferentes tipos de representação na resolução de problemas de divisão. 1993. Dissertação (Mestrado em Educação). Faculdade de Educação, UFPE, Recife.

VIEIRA, E. Intervenção psicopedagógica na fase de representação mental em resolução de problemas matemáticos. 1999. Tese (Doutorado em Educação). Faculdade de Educação, PUC, Porto Alegre, RS.

WERTHEIMER. J. Productive thinking. Chicago: University Press, 1945.

DATA RECEBIMENTO: 05/09/2006

DATA APROVAÇÃO: 07/05/2007 